\title{
Development of Visible Spectroscopic Diagnostics for monitoring Plasma Facing Components impurity sources in the WEST Tokamak
}

\author{
O. Meyer ${ }^{1}$, JC. Giacalone, JY. Pascal, D. Raulin, MH. Aumeunier, C. Gil, \\ S. Larroque, F. Micolon, P. Moreau
}

CEA IRFM

F-13108 Saint-Paul-Lez-Durance, France

E-mail: olivier.meyerlcea.fr

\begin{abstract}
The present work concerns the ongoing development of the visible spectroscopic diagnostic in the framework of the WEST (Tungsten-W Environment in Steady state Tokamak) project that aims at equipping the existing Tore Supra device with a Tungsten divertor in order to test actively cooled Tungsten Plasma Facing Components (PFC) in view of preparing ITER operation [Bucalossi11]. The goal of the diagnostic is to measure PFC impurity sources and Deuterium recycling with sufficient spectral, spatial and temporal resolution adapted to the predicted power deposition profiles on the object observed. Three kind of W PFC must be monitored: the antenna/ launcher limiters, the upper and lower divertors and one of the high field side limiters.
\end{abstract}

Regarding time sampling period, we plan to use two acquisition modes (for the spectrometers): a "standard mode" with $5 \mathrm{~ms}$ sampling period and a "high speed mode" with $0.5 \mathrm{~ms}$ sampling period for ELM study. The spectral feature that will be principally used is the W I line at 400.9 $\mathrm{nm}$ and our system will be optimized for this wavelength. Other impurity elements (H I, O I, C I ...) would also need to be monitored.

A total of 240 optical fibres will be connected in a patch panel in the spectroscopic room and dispatched on the detection systems as photomultiplier tubes and filters to monitor the ELMs or imaging spectrometers especially bought for multiviews analysis. Concerning the status of the project, the design part of the antennas views is now over, prototypes of "In Situ" telescopes and optical fibres bundles have been successfully tested and the series is being manufactured for mid-2015. The design of the divertor views that is on progress is also detailed in the paper.

First EPs Conference on Plasma Diagnostics - $1^{\text {st }}$ ECPD

14-17 April 2015,

Villa Mondragone, Frascati (Rome) Italy

${ }^{1}$ O. Meyer 


\section{Introduction}

The present work concerns the ongoing development of the visible spectroscopic diagnostic in the framework of the WEST (Tungsten-W Environment in Steady state Tokamak) project that aims at equipping the existing Tore Supra device with a Tungsten divertor in order to test actively cooled Tungsten Plasma Facing Components (PFC) in view of preparing ITER operation [Bucalossi11]. The goal of the diagnostic is to measure PFC sources and Deuterium (D) recycling with sufficient spectral, spatial and temporal resolutions adapted to the predicted power deposition profiles on the object.

The first part of the paper concerns the description of the physical requirements and the corresponding measurements specifications. The second part presents the technical solutions implemented and the calculations done to estimate the expected efficiency of the overall setup.

\section{Specifications and technical answers}

\subsection{Physical requirements}

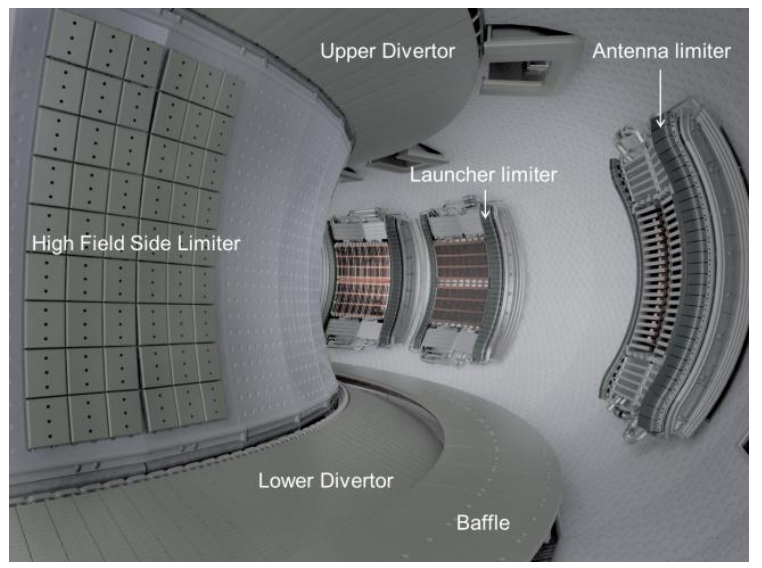

Figure 1: WEST inner view, main PFC components
The aim of the visible spectroscopy system is to permit Plasma Facing Components (PFC) impurity sources estimation as well as incident particle influxes. Regarding sources measurements, all potential impurity need to be monitored $(\mathrm{O}, \mathrm{C} \ldots)$ and namely W. Incident particle influxes will be derived from $\mathrm{D}$ recycling measurement $\left(D_{\alpha}\right)$. The above physical requirements induce constraints on the wavelength range. Another important aspect is the time resolution since the measurements

should permit an estimation of the sources and recycling during ELMs and inter-ELMs periods constituting two different erosion regimes to discriminate. The PFC objects to be monitored shown in figure 1 are the antenna/ launchers limiters, the high field side limiters, the divertors targets and the baffle leading edge. All those PFC are W coated or made of bulk W.

\subsubsection{Antenna / launcher limiters}

The WEST heating schemes will rely on three ion cyclotronic antennas and two lower hybrid launchers. All antennas and launchers W coated limiters will be monitored essentially for operation aspects since it is known that Radio Frequency heating induces W sources that can constitute an operation limitation if transported in the plasma core [Neu13]. In this case the objective will be to monitor the entire limiters surface to detect all the potential $\mathrm{W}$ sources.

\subsubsection{High field side limiters}

In that case, the potential impurity sources are expected only during the ramp up plasma phase where the limiter is in contact with the plasma. During the divertor plasma phases, the distance between the limiter and the separatrix will be larger than $30 \mathrm{~cm}$ as shown on figure 2 
and ASDEX operation feedback taught us that substantial W sources appear when the distance is below $10 \mathrm{~cm}$ [Neu02]. One of the six high field side limiters will thus be monitored essentially during the plasma ramp up phases to detect unexpected high $\mathrm{W}$ influxes during that sensitive phase.
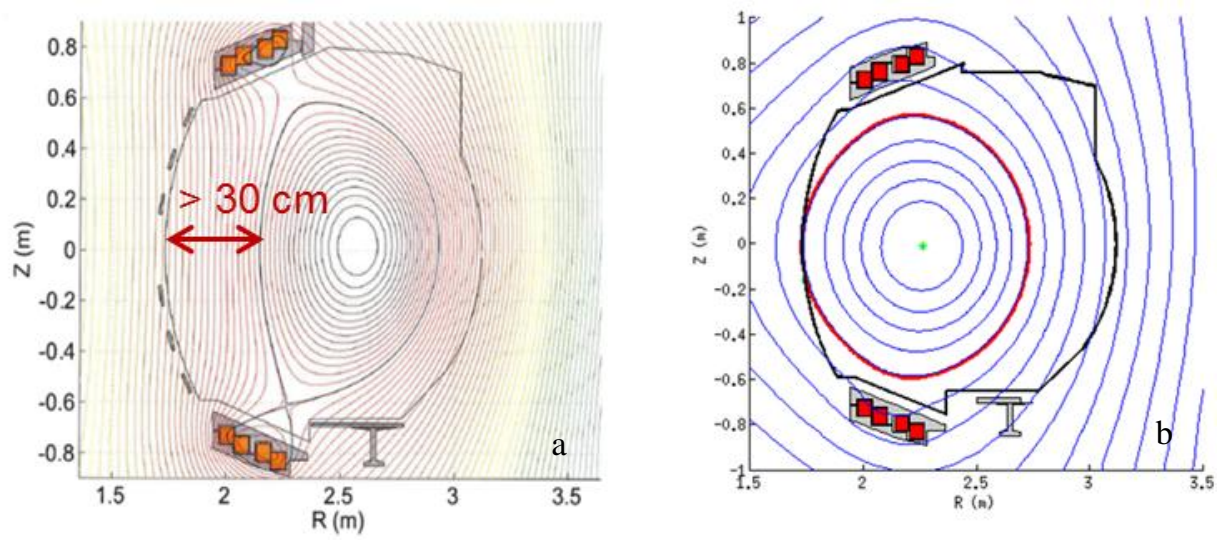

Figure 2: Main flux surfaces during the Xpoint divertor phase (a) and the limiter phase (b)

\subsubsection{Upper and lower $\mathrm{W}$ divertors}

The specifications are the realization of two poloïdal profiles on the lower divertor to monitor the outer and inner strikes points maxima heat flux and the baffle leading edge. One poloïdal profile is planned on the upper divertor to monitor the outer strike point. The expected heat flux deposits and profiles positions are shown on the figure 3.


Figure 3: View of the expected power deposition $\left(\mathrm{MW} / \mathrm{m}^{2}\right)$ on the divertors and baffle. The toroidal modulation period is due to the ripple of the toroidal magnetic field. The 3 profiles to be implemented are represented in green

\subsection{Measurements requirements}

The above physical specifications induce some requirements on the measurements. Regarding the acquisition time, an acquisition mode with $5 \mathrm{~ms}$ sampling period is sufficient for ELMs and Inter-ELMs periods discrimination since the typical Elms expected in WEST present a frequency of about $50 \mathrm{~Hz}$ and $2 \mathrm{~ms}$ duration [Bourdelle15]. Acquisition time below $0.5 \mathrm{~ms}$ is required for ELMs study.

Concerning the wavelength aspects, the spectral feature of interest for the $\mathrm{W}$ is the $\mathrm{W}$ I line at $400.9 \mathrm{~nm}$ and our system will be specifically optimized for that wavelength. Deuterium and 
other impurity elements will also be monitored requesting a spectral coverage available from $400 \mathrm{~nm}$ to $700 \mathrm{~nm}$.

Another critical aspect is the geometry of the lines of sight. The $\mathrm{W}$ being a high reflective material in the visible range, it is very important to favour normal incidence on the observed objects to avoid specular reflections.

\subsubsection{Antenna /launcher limiters}

The limiters are the same for both antennas and launchers and are shown on the figure 1. To fulfil the physical specifications, a spatial resolution of $90 \mathrm{~mm}$ spot size that corresponds to the antenna limiter width is required. A total of 12 lines of sight are necessary to cover the full antenna limiter.

\subsubsection{High field side limiter}

Given the physical specifications, the plan is to set up a poloidal profile around the centre of the limiter where the plasma is in contact during the ramp up phases (figure 2). Smooth power deposit flux profile being expected in the high field side area, a spatial resolution of 70 $\mathrm{mm}$ with 12 lines of sight has been retained covering $\pm 420 \mathrm{~mm}$ around the mid plane.

\subsubsection{Upper and lower divertors}

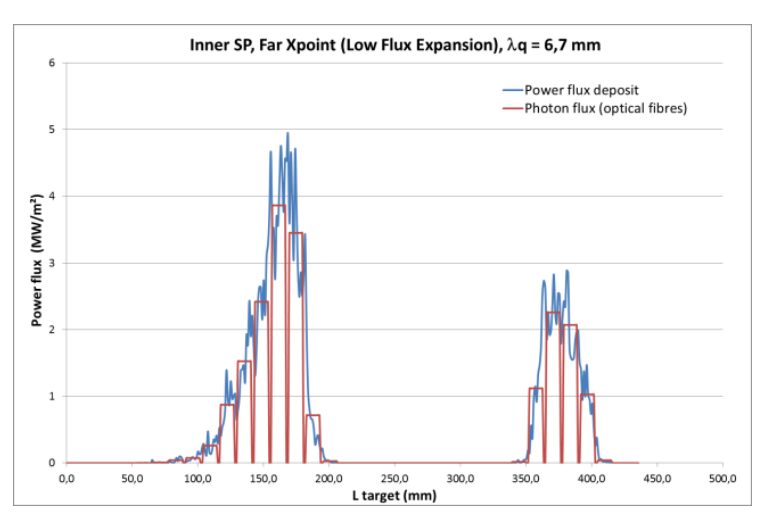

Figure 4: simulation of the source profile measured in the case of a peaked power flux deposit. The blue curve is the power flux $\left(M W / \mathrm{m}^{2}\right)$, the red curve is the photon flux collected by the optical fibres (arbitrary units), $L$ is the distance along the target
The physical specifications for the divertors views impose strong constraints on the spatial resolution since the power flux deposit profile is expected to be peaked as shown in the figure 3 . A spatial resolution of $10 \mathrm{~mm}$ has been foreseen to be sufficient to fulfil the physical specification and namely estimate the impurity sources profile decay length. The figure 4 shows calculation of the profiles obtained by using $10 \mathrm{~mm}$ spatial resolution for the WEST low flux expansion scenario. The hypothesis here is that the sources profile is proportional to the power influx deposit that is often verified for heavy impurities like $\mathrm{W}$ but less for light impurities since the longer ionization length smoothes the profiles. The calculations show that the $10 \mathrm{~mm}$ spatial resolution is sufficient for a good estimation of the impurity influx profiles and namely the decay length. A total number of 36 lines of sight will thus be implemented for each profile.

\section{Technical solutions implemented}

The visible spectroscopy diagnostic is consists of 240 lines of sight materialized by optical fibres. In the overall system that will be implemented, the signals are transported using optical fibres and connected to a patch panel to be dispatched on the various detection systems that will be available in the spectroscopy room. 


\subsection{Optomecanical design}

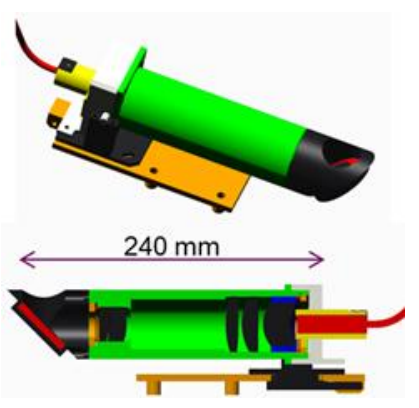

Figure 5: Divertor telescope equipped with a relay mirror

To respect the specifications and namely the normal incidence, we opted for optics directly installed in the vacuum chamber. Dedicated telescopes setup have been designed to image the monitored PFC on $400 \mu \mathrm{m}$ diameter optical fibres mounted in slit assembly. Those telescopes (figure 5) are composed of 5 or 6 fused silica lenses depending on the view and the support design permits a 3 rotation axis tuning. The telescopes are connected to optical fibres bundles of 6, 12 or 36 fibres for antenna limiters, high field side limiter and divertors views respectively. The optical fibres bundles contain a vacuum feedthrough that is directly welded on dedicated flange. Optical fibres from bundles are then connected to 50 meters optical fibres links and connected to the patch panel in the spectroscopy room.

\subsection{Integration in Tokamak harsh environment}

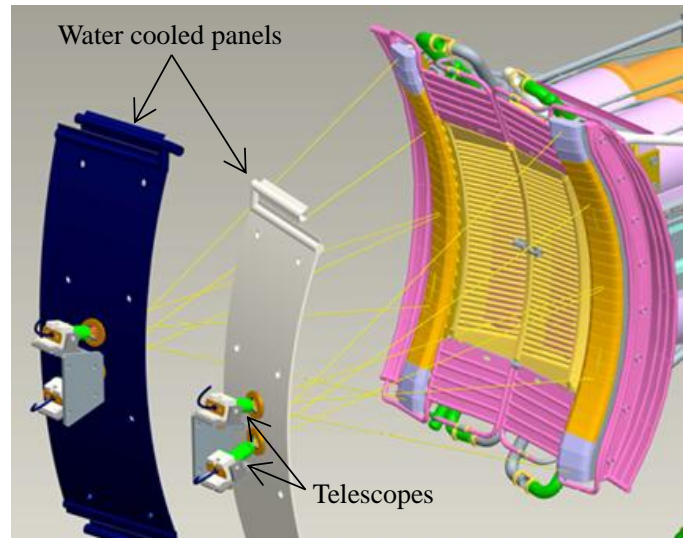

Figure 6: Antenna limiter telescopes

Choosing an in vessel optical assembly required adapting the objects to the constraining Tokamak environment. All the materials must be vacuum compatible, sustain $200^{\circ} \mathrm{C}$ baking temperatures and $0.14 \mathrm{MW} / \mathrm{m}^{2}$ of power deposit on long pulse duration. To sustain the power flux deposit, all the components are protected behind actively cooled thermal shields.

The antenna limiters telescopes are implemented directly behind the high field side water cooled panels and a fused silica window that permits to keep the first lens below $120^{\circ} \mathrm{C}$ that is admissible for the system (figure 6).

The divertors telescopes, shown in figure 5 are protected by dedicated actively cooled panels and a relay mirror but required dedicated studies since presenting a notable radial extension in the vacuum vessel (figure 7). The potential risks regarding the plasma power fluxes have been studied. The ripple is not an issue since being not expected at the low field side. Fast

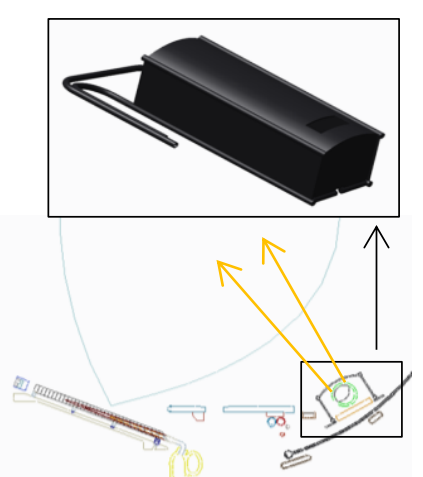

Figure 7: Implantation of the upper divertor view. View of the actively cooled thermal shield. particle fluxes are also not probable due to the distance of $24 \mathrm{~cm}$ between the thermal shield and the separatrix. The higher risk estimated is a vertical displacement (VDE) during which the plasma would go in contact with the telescope thermal shield. We thus introduced a new constraint on the radial extension of the system that is to avoid contact with a $50 \mathrm{~cm}$ minor radius plasma.

Regarding the optical fibres bundles, all of them are protected by PTFE tubing that will be also protected from plasma radiated power by cooling plates welded on water 
system pipes (design currently in progress at the time of the paper submission)

\subsection{Detection systems}

Our detection systems setups will contain both grating spectrometers connected to Coupled Charge Device (CCD) cameras and Photomultiplier Tubes (PMT) associated with interference filters systems. The former systems allow multiviews wavelength resolved measurements that namely permits fine background subtraction and line profile study. The later systems authorises high dynamics fast acquisitions.

Concerning the grating spectrometer setups, they will be dedicated in priority to measure the W I line at $400.9 \mathrm{~nm}$. The instrumental constraints are the efficiency at $400 \mathrm{~nm}$, the multiviews capability (maximizing the number of optical fibres connected on one camera) and a spectral resolution below $2 \mathrm{~A}$ to avoid interfering lines (Ar II at $401.29 \mathrm{~nm}$ for example). We choose the Isoplane imaging spectrometer developed by Princeton Instrument that presents an $\mathrm{f}$ number of 4.6 and permits to simultaneously treat 27 optical fibres vertically aligned in front of the entrance slit without any crosstalk. The spectrometer is connected to a Proem CCD camera presenting a square ship of 1024 x 1024 pixels of $13 \mu \mathrm{m}$, a quantum efficiency of $75 \%$ at 400 $\mathrm{nm}$ and $10 \mathrm{MHz}$ readout capabilities. The spectrometers are equipped with $600 \mathrm{gr} / \mathrm{mm}$ and $1200 \mathrm{gr} / \mathrm{mm}$ gratings. The main performances obtained with the whole setup are detailed in table 2 and permit to meet the specifications.

Table 1: Main performances of the spectrometers instrumental setup

\begin{tabular}{|l|l|l|l|l|}
\hline $\begin{array}{l}\text { Grating } \\
(\mathrm{gr} / \mathrm{mm})\end{array}$ & $\begin{array}{l}\text { FWHM } \\
(\AA)\end{array}$ & $\begin{array}{l}\text { Spectral coverage } \\
(\AA)\end{array}$ & $\begin{array}{l}\text { Standard time resolution } \\
(\mathrm{ms})\end{array}$ & $\begin{array}{l}\text { Fast time resolution } \\
(\mathrm{ms})\end{array}$ \\
\hline 600 & 1 & 660 & 5 for 27 views & 0.5 for 1 view \\
\hline 1200 & 0.5 & 330 & & \\
\hline
\end{tabular}

The PMT systems will be dedicated to ELMs studies that require very fast acquisition. A system equipped with $\mathrm{H}_{\alpha}$ filters will permit ELM resolution with a target sampling period of 25 $\mu$ sec. Another system in study in the framework of the Eurofusion contact at the moment of writing the paper concerns the implementation of a PMT based polychromator system. It would permit the study of several spectral lines on the same line of sight that is very interesting namely for the ELMs induced sputtering yield determination.

\subsection{Estimation of the photon flux collected by a spectrometer setup}

Even if the predominant parameter of choice of each part of the system was the efficiency at $400 \mathrm{~nm}$, an estimation of the collected flux was necessary to confirm our system meets the time specifications. The table 2 shows the transmission of each major interface.

Table 2: Transmission (\%) of each critical system interface

\begin{tabular}{|c|c|}
\hline $\begin{array}{l}\text { Optical fibre links } \\
50 \mathrm{~m} \text { (typical attenuation } 40 \mathrm{~dB} / \mathrm{km} \text { ) }\end{array}$ & \multirow{2}{*}{60} \\
\hline $\begin{array}{l}2 \mathrm{FCPC} \text { connections } \\
\text { (typical loss } 0.15 \mathrm{~dB} \text { ) }\end{array}$ & \\
\hline Spectrometer coupling (F-numberfibre 2 (F-numberspectro ${ }^{2}$ ) & 24 \\
\hline
\end{tabular}




\begin{tabular}{|l|l|}
\hline $\begin{array}{l}\text { Slit shadowing } \\
\text { (Slit surface/ Fibre optics surface) }\end{array}$ & 63 \\
\hline Grating transmission at $400 \mathrm{~nm}$ & 75 \\
\hline Overall transmission & $\mathbf{7}$ \\
\hline
\end{tabular}

If we refer to ASDEX and JET experimental campaigns, the expected radiance for the W I line is between $10^{16}$ and $10^{19} \mathrm{Ph} /\left(\mathrm{m}^{2}\right.$.s.sr $)$. In the case of the antenna limiter views which telescopes present f-number of 2.6, the photon flux F collected by the system is determined by the expression $\mathrm{F}=\mathrm{L} \times \mathrm{G}$ where $\mathrm{L}$ is the radiance and $\mathrm{G}$ the geometrical etendue. In our case, the geometrical etendue is the product of the collection solid angle of the telescope $(0.116 \mathrm{sr})$ and the surface of the collecting fibre optics $\left(0.126 \mathrm{~mm}^{2}\right)$, the resulting geometrical etendue is $\mathrm{G}=$ 1.5.10 $0^{-8} \mathrm{~m}^{2}$.sr. The photon flux collected by the fibre optics is thus $1.5 .10^{8} \mathrm{Ph} / \mathrm{s}$ and the flux received by the CCD camera $1.5 .10^{8} \times 0.07 \sim 10^{7} \mathrm{Ph} / \mathrm{s}$. The spectral line will spread on about 15 pixels meaning about $7.10^{6} \mathrm{Ph} / \mathrm{s}$ collected on a single pixel (all the pixels of the fibre will be summed vertically before reading). Around 4000 photons should at minima be collected by the CCD pixel during a $5 \mathrm{~ms}$ acquisition time, which is sufficient for such type of camera. In the case of $0.5 \mathrm{~ms}$ acquisition time, a binning between different lines of sight will be required to collect sufficient fluxes but we will probably have to rely on the PMT based system for that part.

\section{Conclusion}

The paper detailed in a first part the physical requirements and the corresponding measurements specifications for the WEST visible spectroscopy system that will be dedicated to Plasma Facing Component sources assessment and namely W. The second part of the paper showed that the technical solutions implemented meet the specifications and an estimation of the photon flux collected by the spectrometer setups permits namely to show that the system would be sufficiently efficient for our application. Concerning the current status of the project at the moment of the paper submission, the antenna views system is being manufactured when the design studies for the lower divertor and the high field side limiter views are still in progress and should be over in two months.

\section{Acknowledgment}

This work has been carried out within the framework of the EUROfusion Consortium and has received funding from the Euratom research and training programme 2014-2018 under grant agreement No 633053. The views and opinions expressed herein do not necessarily reflect those of the European Commission.

\section{References}

[Neu02] R. Neu et all, Plasma Phys. Control. Fusion 44, 811-826 (2002)

[Neu13] R. Neu et al., J. Nucl. Mater. (2013)

[Bucalossi11] J. Bucalossi et al., Fusion Engineering and Design 86, 684-688 (2011)

[Bourdelle15] C. Bourdelle et al., Nucl. Fusion 55, 063017 (2015) 